\title{
Development of a transient expression assay for detecting environmental oestrogens in zebrafish and medaka embryos
}

Okhyun Lee, Charles R Tyler ${ }^{*}$ and Tetsuhiro Kudoh*

\begin{abstract}
Background: Oestrogenic contaminants are widespread in the aquatic environment and have been shown to induce adverse effects in both wildlife (most notably in fish) and humans, raising international concern. Available detecting and testing systems are limited in their capacity to elucidate oestrogen signalling pathways and physiological impacts. Here we developed a transient expression assay to investigate the effects of oestrogenic chemicals in fish early life stages and to identify target organs for oestrogenic effects. To enhance the response sensitivity to oestrogen, we adopted the use of multiple tandem oestrogen responsive elements (EREC38) in a Tol2 transposon mediated Gal4ff-UAS system. The plasmid constructed (pTol2_ERE-TATA-Gal4ff), contains three copies of oestrogen response elements (3ERE) that on exposure to oestrogen induces expression of Gal4ff which this in turn binds Gal4-responsive Upstream Activated Sequence (UAS) elements, driving the expression of a second reporter gene, EGFP (Enhanced Green Fluorescent Protein).

Results: The response of our construct to oestrogen exposure in zebrafish embryos was examined using a transient expression assay. The two plasmids were injected into 1-2 cell staged zebrafish embryos, and the embryos were exposed to various oestrogens including the natural steroid oestrogen $17 ß$-oestradiol $\left(E_{2}\right)$, the synthetic oestrogen 17a- ethinyloestradiol ( $\left.\mathrm{EE}_{2}\right)$, and the relatively weak environmental oestrogen nonylphenol (NP), and GFP expression was examined in the subsequent embryos using fluorescent microscopy. There was no GFP expression detected in unexposed embryos, but specific and mosaic expression of GFP was detected in the liver, heart, somite muscle and some other tissue cells for exposures to steroid oestrogen treatments ( $E_{2} ; 10 \mathrm{ng} / \mathrm{L}, \mathrm{E}_{2} ; 100 \mathrm{ng} / \mathrm{L}$, after $72 \mathrm{~h}$ exposures). For the NP exposures, GFP expression was observed at $10 \mu \mathrm{g} \mathrm{NP/L}$ after $72 \mathrm{~h}$ (100 $\mu \mathrm{g} \mathrm{NP/L}$ was toxic to the fish). We also demonstrate that our construct works in medaka, another model fish test species, suggesting the transient assay is applicable for testing oestrogenic chemicals in fish generally.
\end{abstract}

Conclusion: Our results indicate that the transient expression assay system can be used as a rapid integrated testing system for environmental oestrogens and to detect the oestrogenic target sites in developing fish embryos.

Keywords: Oestrogenic endocrine disrupting chemicals, Oestrogen response elements, UAS-GAL4, Transient expression assay, Zebrafish

\footnotetext{
*Correspondence: c.r.tyler@exeter.ac.uk; t.kudoh@exeter.ac.uk

Biosciences, College of Life and Environmental Sciences, University of Exeter, Exeter, Devon EX4 4PS, UK
}

\section{Biomed Central




\section{Background}

Decades of research have shown that a number of natural and man-made chemicals interfere with the endocrine system and can result in adverse health effects in humans, mammals and fish [1-3]. Wildlife living in, or closely associated with the aquatic environment have been shown to be especially impacted by these so-called endocrine-disrupting chemicals (EDCs), because our freshwaters, estuaries and oceans act as sinks for chemical discharges [4]. Oestrogenic EDCs in the environment are of particular concern. Endogenous oestrogens are a group of closely related steroid hormones essential in the development and functioning of the reproductive system. Extensive work has been conducted on natural (e.g. $17 \beta$-oestradiol) and synthetic oestrogens (e.g. $17 \alpha-$ ethinyloestradiol from the contraceptive pill) and their interactions in the vertebrate body, including their tissue distributions, mechanisms of action and pathways of elimination [5-7]. Furthermore, adverse effects of exposure to environmental oestrogens have received considerable research attention in a number of animal species, but this work is largely restricted to adults and juveniles $[6,8]$, and there has been little study of effects on embryos. Nothing is known regarding the relative sensitivities of the different cell types and tissues to oestrogenic EDCs, or other contaminants, during embryogenesis. Furthermore, in spite of the widespread concern for EDCs in the aquatic environment, there are very few bioassay systems that are sufficiently sensitive for accurate prediction of adverse biological effects. Moreover, conventional methods of EDCs detection such as tissue culture [9] and in vitro techniques [10,11] are limited in their capacity to elucidate oestrogen signalling pathways and tissue specific physiological impacts.

Teleost fish have three oestrogen receptors (ER), ERalpha, ERbeta-1 and ERbeta-2, that show tissue specific patterns of expression and function in adults [12-14]. The different ER subtypes are also widely expressed in body tissues in early life stages, from embryos to young larvae [15], suggesting crucial roles of these signalling pathways in early development. Indeed, recently it was found that knockdown of ER-beta2 in the zebrafish suppressed normal development of the lateral line neuromast cells [16]. Endogenous oestrogen receptors activated by oestrogenic chemicals bind to oestrogen response elements within regulatory regions of oestrogenresponsive genes. Response elements are recognized by nuclear transcription factors, including members of the steroid/nuclear receptor super family that then, together with various other regulatory factors mediate transcription of the associated downstream genes [17].

The adopted model in this work, the zebrafish (Danio rerio) has become one of the most commonly used animals for examining effects of aquatic pollutants [18].
Furthermore, with the available genomic resources and suitability of this species for molecular manipulations, the zebrafish has been applied more widely for research in developmental biology and understanding of disease processes. The medaka (Oryzias latipes) is another model species widely used in ecotoxicology research and for the development of transgenic techniques. Many studies have shown that early life stages of fish have the greatest sensitivity to environmental contaminants and effects on development in the zebrafish and medaka are greatly facilitated by the fact that their embryos are transparent. [19-21].

Use of tissue-specific promoters has become a powerful tool for studies on endogenous gene expression [22] and to analyse the function of promoters [9,23-25] and transgenic fish have become an established technique in developmental analyses. This generally includes using a specific promoter and a green fluorescent protein (GFP) or a luciferase reporter gene. To improve the efficiency of the sensitivity, tissue specificity and ease of generating transgenic fish, various manipulated gene systems have recently been introduced. One of these is the Gal4-UAS system. This is now used widely for the over expression of transgenes in various transgenic animals, including zebrafish [26]. This system comprises a two-part expression system that utilises the yeast transcription activator protein Gal4 and its target sequence UAS (Upstream Activated Sequence), to which Gal4 binds to activate gene transcription $[27,28]$. The Tol2 transposon system, originally identified in the medaka [29], has recently been used to enhance the success rate of DNA integration into the zebrafish genome [30,31]. This is illustrated in the work of $[30,32]$ ) where used of Tol2 increased the transgenesis rate of linear DNA from 5\% to $50 \%$.

Transgenic zebrafish have considerable potential for use in aquatic ecotoxicology to screen and test for hormone mimics and potentially to develop a more advanced system for assessing health impacts of chemicals. As a consequence there has been considerable activity in a number of laboratories to develop transgenic zebrafish as tools for screening and testing of chemicals [33-36]. Transgenic fish have the advantage that tissue specific effects of EDCs can be identified to allow for more directed and detailed studies to inform on health outcomes. However, it is time consuming, both to produce and maintain the stable transgenic lines. As a consequence, a number of studies have investigated the use of transient expression assays to examine the spatial and temporal expression of reporter genes that are fused to the regulatory regions of various genes in zebrafish embryos [37,38]. To date, biosensor transgenic fish (TG fish) have only been generated in the zebrafish and medaka, and such technology has not been applied widely to other fish species. In theory, however, having 
developed the technology for these model species, it would be possible to develop a functional 'transient assay', by which vector DNA is transiently introduced into the fish embryo, in almost any fish species where the eggs produced can physically be injected, and thus examine the effect of chemical exposure specifically in those fish species.

To date, there have not been any reported transient expression assays for the detection of oestrogenic EDCs. The transient expression assay process normally involves the injection of fertilised embryos with a construct and followed by assaying of the response (e.g. GFP or Luciferase) once the embryos/larvae have reached the desired stage of development. A major advantage of the transient assay would be that the technique is applicable to a variety of fish species for examining tissue, stage and chemicals specific responses without the need to generate TG fish lines.

With a plan to develop a rapid and sensitive transient expression assay system to evaluate the oestrogenic activity of environmental chemicals in different fish embryos, we utilised a Gal4ff-UAS system that incorporates a two-step signal amplification process and a synthetic oestrogen responsive element with 3EREs. A major aim in this initial work was to make a construct that was highly responsive to oestrogenic EDCs. We then examined the responsiveness of the oestrogen response elements and tissue and stage specific responses in zebrafish using a transient expression assay. Finally, we tested our plasmid in another fish species, the medaka, to show that transient expression assay is suitable for observation of effects of oestrogenic compounds in other fish test species.

\section{Results and discussion}

\section{Construction of reporter gene vectors with oestrogen} responsive promoters

In the sequence of results reported, we first developed a novel transient assay system using a synthetic oestrogen responsive element, Tol2 and Gal4-UAS systems and the GFP reporter gene, responsive to environmental oestrogen. We then investigated the functional capability of this construct and response to a range of potent and weak environmental oestrogens using a transient expression assay using green fluorescent microscopy in zebrafish embryos/larvae. Finally, we proofed our vector system further in another fish species, the medaka.

The construct produced for detecting oestrogen is shown in Figure 1. Specific primers of EREc38 were run in PCR reactions to produce the EREs. The EREs were 38 base pair (bp) sequences that consisted of a 17-bp inverted repeat 5'-CAGGTCA nnn TGACCTG-3' followed by an AT-rich sequence [39]. Other studies that have made TG fish for screening of oestrogen chemicals have used various (and different) promoters, including for the following genes, vitellogenin (VTG) [34], choriogenin L (ChgL) [40] and aromatase B [41]. Legler et al. (2000) [42] developed a transgenic zebrafish line that expressed the reporter gene luciferase (LUC) for examining oestrogenic compounds. Although the construct they used, pEREtata-LUC, was sensitive, luciferase is not as stable as GFP. The luciferase half-life varies from 3 to

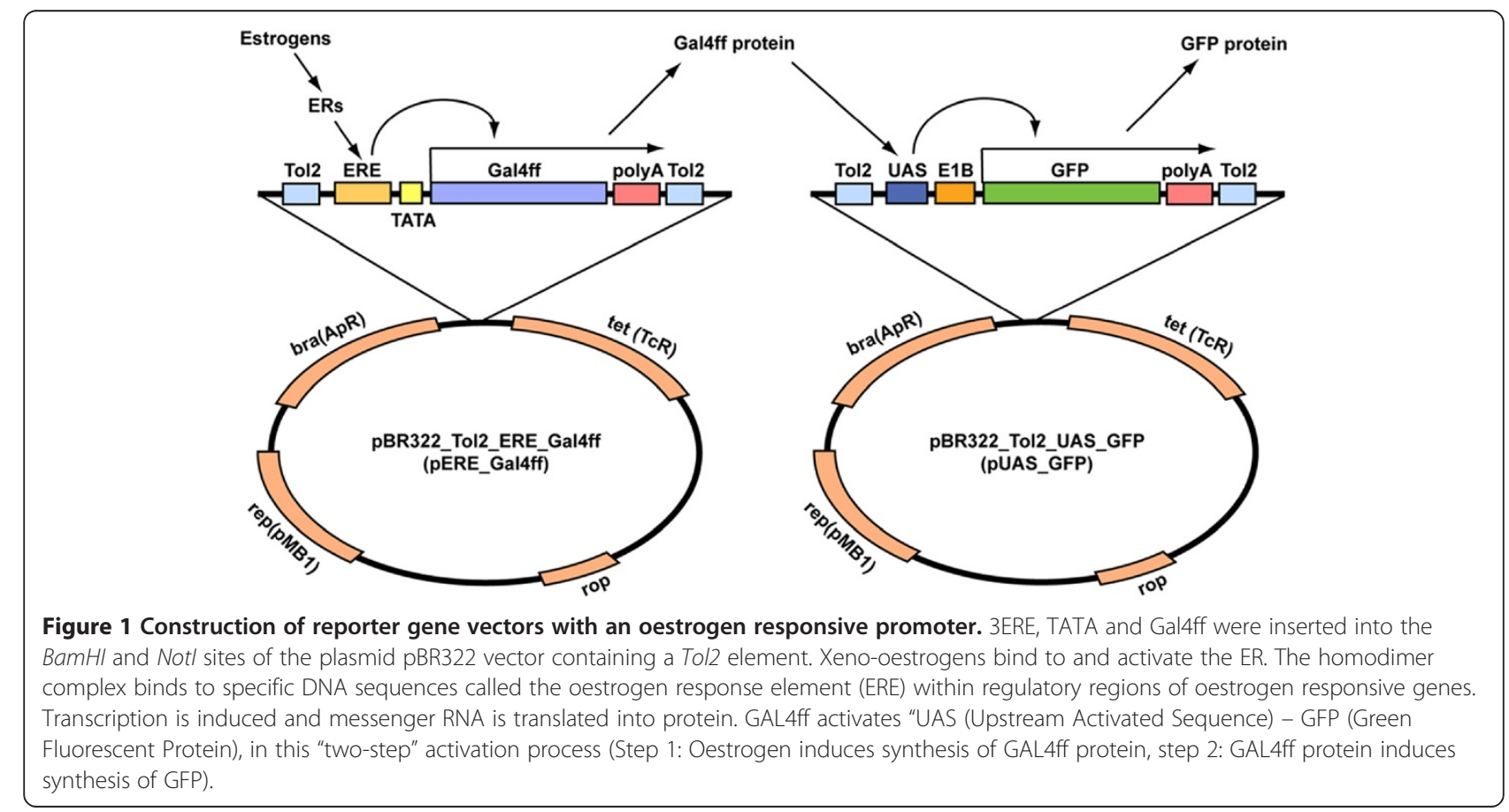


$14 \mathrm{~h}$ [43], as it strongly depends on the host cell and the gene construct. Furthermore, measurement of luciferase is a bioassay that requires tissue homogenisation. In contrast, GFP can be detected in living cells/organisms. The use of GFP in intact fish also has advantages over use of in vitro transfected cell cultures, because it allows for integration of toxicodynamic and toxicokinetic factors into the response [44]. The constructs that have been used in previous studies for developing oestrogen response systems, such as pEGFP-ChgL [45], pZVTG1-EGFP [34] are also liver specific genes.

In a mammalian cell culture system, Sathya et al. (1997) [39] showed that a construct with 3EREs produced an enhanced expression compared with 2EREs and 4EREs, especially in response to the natural oestrogen, oestrdaiol-17 $\beta\left(\mathrm{E}_{2}\right)$. We therefore similarly adopted three copies of ERE in our construct. For the transcriptional initiation, the TATA box sequence was inserted at 158 bp upstream of the Gal4ff start codon and the Tol2 transposon system was adopted to provide a more efficient transient reporter analyses. After the sequential synthesis of five successive constructs, we generated a novel plasmid vector system, p3ERE-TATAGal4ff containing, Tol2 and a poly A tail and employing two steps for amplification of the signal (Figure 1) where Gal4ff activates "UAS-GFP'. In the activation process, the first step, binding of oestrogens to ER and subsequent binding to ERE, induces the synthesis of GAL4ff protein and in the second step Gal4ff protein induces synthesis of GFP. This two-step process allows amplification of the resulting signal which, in theory, should give rise to a more "sensitive" transient expression assay system for detecting oestrogen chemicals than has been developed previously. In our approach, we used Gal4ff rather than the more commonly used Gal-VP16/UAS system, because GAL4ff is reported to be less toxic than GAL4-VP16 in zebrafish cells [46].

\section{Induction of GFP in the zebrafish embryo transient expression assay}

In the transient microinjection studies, expression of gene in zebrafish and medaka embryos was mosaic because the microinjected recombinant DNAs were not integrated to all cells. We minimised this problem by flanking the transgene with a Tol2 transposon and we were then able to identify target tissues for oestrogens in early life stages more effectively [31].

No differences in mortality rate were observed between both control and oestrogen chemical treated groups ( $>95 \%$ survival), with the exception for $100 \mu \mathrm{g}$ $\mathrm{NP} / \mathrm{L}$ where the most embryos died by $24 \mathrm{hpf}$. The lack of toxicity effects in zebrafish embryos at the exposure concentrations adopted (with the exception of $100 \mathrm{ug}$ NP/L) compared very favourably with previously reported findings (e.g. Scholz and Gutzeit (2000) for $\mathrm{EE}_{2}$ [47] and Kishida et al. (2001) for $E_{2}$ [48]).

GFP expression was seen at $24 \mathrm{~h}$ after exposure to $\mathrm{EE}_{2}$ (data not shown), albeit at low level, whereas there was no GFP expression detected in the controls at this time. There was stronger and more specific tissue GFP expression in $\mathrm{EE}_{2}$ treated embryos at 72 and $96 \mathrm{hpf}$, focused mainly in the liver, muscle and heart and this was therefore adopted as the time period for assessing tissue specific effects of oestrogens in the transient assay.

At 72 and $96 \mathrm{hpf}$ there was no GFP expression detected in embryos in the unexposed controls (Figure 2, Ai-Aiii, Additional file 1: Figure S1), but GFP expressing cells were seen clearly after exposure to $\mathrm{EE}_{2}$ (Figure 2, $\mathrm{Ci}-D i i i$, Additional file 1: Figure Bi-Bii). No specific GFP expression was observed in the liver at the lowest exposure concentration of $\mathrm{EE}_{2}(10 \mathrm{ng} / \mathrm{L})$, but weak GFP expression signals were detected in the heart and somite muscle for this exposure concentration (Figure 2, Bi-Biii). GFP expression was most clearly seen in the higher $\mathrm{EE}_{2}$ exposures. For exposure to $100 \mathrm{ng} \mathrm{EE}_{2} / \mathrm{L}$ GFP was detected in a feint and dispersed manner in the liver and there was strong GFP expression in the heart and somite muscle. GFP expression in the heart was confirmed by the periodical contractile movement of the GFP expressing cells with each heart beat in the live injected larvae. Strong GFP expression was also observed in the anterior

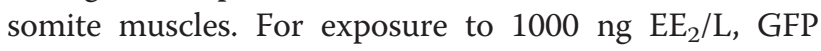
expression was induced strongly in the liver, somite muscle and heart (Figure 2, Ci-Ciii). Moreover, many GFP expressing cells were observed in the anterior lateral part of the injected embryos (Figure 2, Di). The number of cells expressing GFP appeared to be positively associated with the concentration of the $E_{2}$ exposure (Table 1). For the transgenic lines of zebrafish that have now been developed, it has bee shown that oestrogenic EDCs activate ERE in liver [34,42], forebrain [41], neuromast [49], heart [36,49] and somite muscle [49] and this is consistent with the expression patterns seen here our transient assay.

Exposure to the natural steroid oestrogen, $\mathrm{E}_{2}$, resulted in similar responses to that seen for $E_{2}$. No GFP expression was observed in unexposed controls (Figure 3, Ai-Aiii). Similarly, no GFP expression was observed for the lowest $E_{2}$ exposure concentration (10 ng $E_{2} / L$, data not shown). No GFP expression was observed in the liver at $100 \mathrm{ng} \mathrm{E}_{2} / \mathrm{L}$, but it was seen in the muscle (somite and cranial) and heart (Figure 3, Bi-Biii). Exposure to $1000 \mathrm{ng} \mathrm{E}_{2} / \mathrm{L}$ strongly induced GFP expression in the heart, otic vesicle and somite muscle and there were some GFP expressing cells observed in the liver (Figure 3, Ci-Ciii). With $E_{2}$, as well as with $\mathrm{EE}_{2}$, the GFP signal was also observed in the lateral line neuromast cells albeit a lower frequency (Additional file 1: Figure S1, 


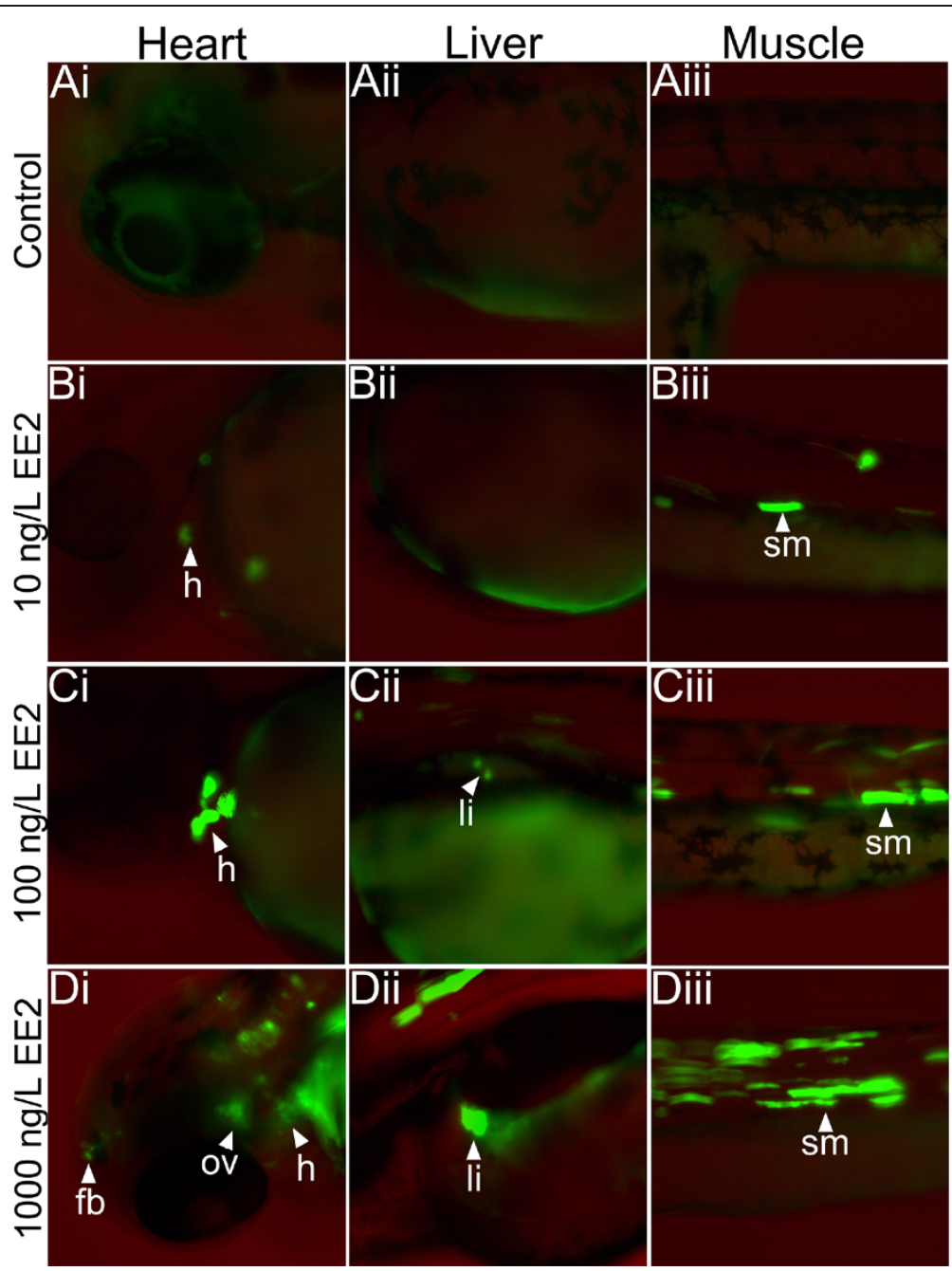

Figure 2 Expression of GFP in zebrafish embryos in the transient expression assay using the mixture of the plasmid pERE-Gal4ff containing the Tol2 transposase mRNA and the plasmid pUAS-GFP. Fertilised zebrafish eggs were microinjected at the $1-2$ cell stages with the mixture of two plasmids. Injected embryos were then exposed for up to 72 hour post-fertilisation (hpf) to 17a-ethinyloestradiol (EE $E_{2}$. These embryos showed specific mosaic expression of the reporter. The injected embryos exposed to 1000, 100 and $10 \mathrm{ng} / \mathrm{L}$ of EE 2 expressed GFP in cells most strongly in the somite muscle, heart and liver (72 hpf). This experiment was run in duplicate and was repeated at least seven times.

Table 1). Exposure to $10 \mu \mathrm{g} \mathrm{NP/L}$ induced low level GFP expression in the heart and somite muscle (Figure 3, DiDiii). The reason for weak GFP induction by NP might be due to a lower sensitivity of early development stages to this chemical in the zebrafish, as has been suggested previously [50]. In addition, it has been reported that NP might be transformed in fish to less potent metabolites $[51,52]$. These data indicate that different xeno-

Table 1 Percentage of plasmid injected zebrafish expressing GFP in different body tissues after exposure to oestrogens (at $72 \mathrm{hpf}$ )

\begin{tabular}{|c|c|c|c|c|c|c|c|}
\hline & \multirow[t]{2}{*}{ Control } & \multicolumn{3}{|c|}{$\mathrm{EE}_{2}$ (ng/L) } & \multicolumn{2}{|c|}{$E_{2}$ (ng/L) } & \multirow{2}{*}{$\begin{array}{c}\mathrm{NP}(\mu \mathrm{g} / \mathrm{L}) \\
10\end{array}$} \\
\hline & & 1000 & 100 & 10 & 1000 & 100 & \\
\hline Liver & 0 & $74.3 \%(223 / 300)$ & $61.6 \%(185 / 300)$ & 0 & $70.7 \%(212 / 300)$ & $18.7 \%(56 / 300)$ & 0 \\
\hline Heart & 0 & $82.7 \%(248 / 300)$ & $66.3 \%(199 / 300)$ & 0 & $78 \%(234 / 300)$ & $65 \%(195 / 300)$ & $56 \%(168 / 300)$ \\
\hline Muscle & $13.3 \%(40 / 300)$ & $90.3 \%(271 / 300)$ & $78.3 \%(235 / 300)$ & $27.3 \%(82 / 300)$ & $87 \%(261 / 300)$ & $74 \%(222 / 300)$ & $71.3 \%(214 / 300)$ \\
\hline Forebrain & & $33 \%(99 / 300)$ & 0 & 0 & $25.6 \%(77 / 300)$ & 0 & 0 \\
\hline Otic vesicle & $19 \%(47 / 300)$ & $26.3 \%(78 / 300)$ & $16.6 \%(50 / 300)$ & $10.6 \%(32 / 300)$ & $27 \%(81 / 300)$ & $25.3 \%(76 / 300)$ & 0 \\
\hline lateral line neuromast & $10 \%(32 / 300)$ & $22.3 \%(67 / 300)$ & $13 \%(39 / 300)$ & $14.7 \%(44 / 300)$ & $25.3 \%(76 / 300)$ & $18 \%(54 / 300)$ & $4 \%(12 / 300)$ \\
\hline
\end{tabular}




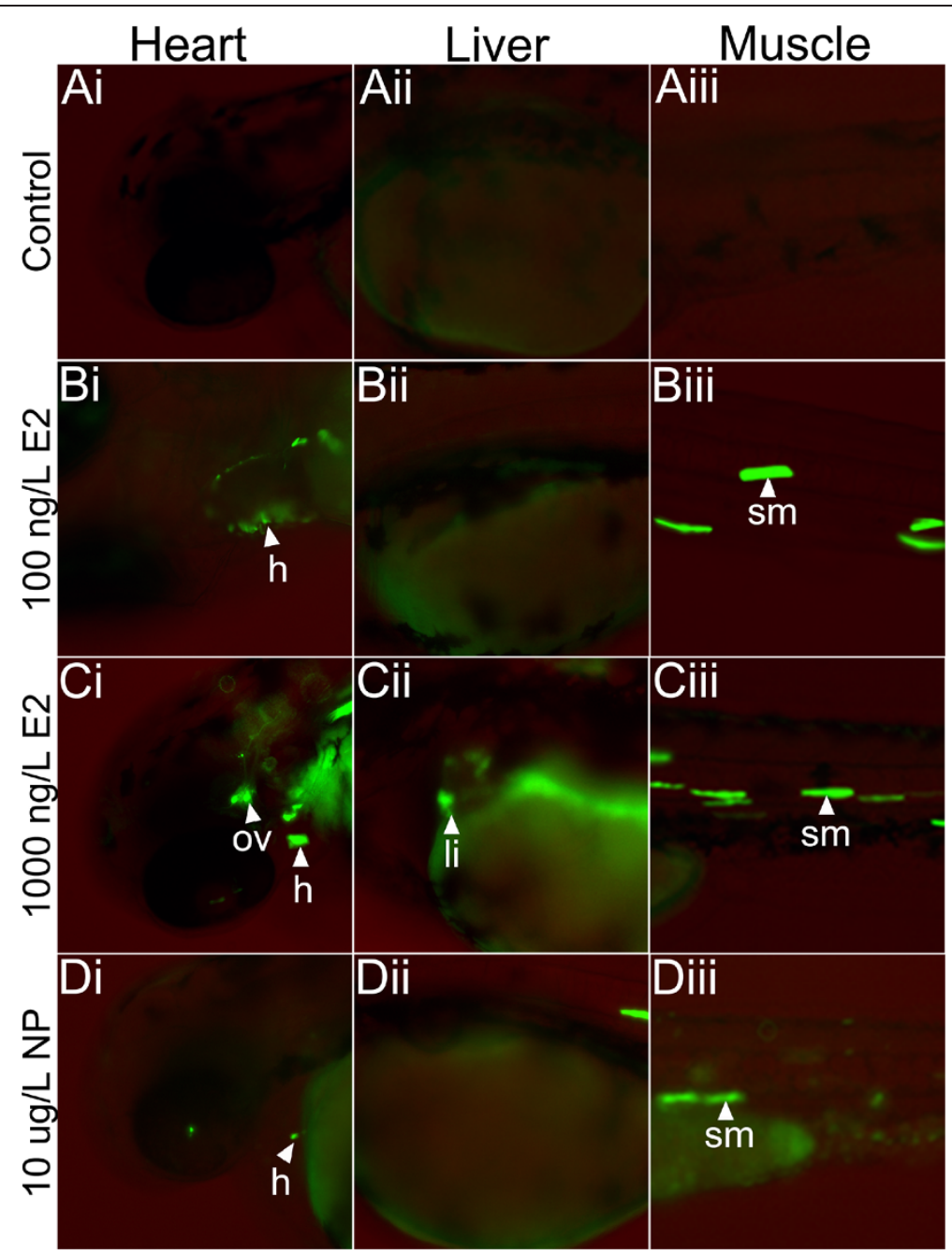

Figure 3 Comparison of detectable response concentrations for different oestrogen compounds in the zebrafish embryo transient assay. Injected embryos were exposed for up to $72 \mathrm{~h}$ post-fertilisation to $17 \beta$-oestradiol $\left(\mathrm{E}_{2}\right)$ and nonylphenol (NP) and fluorescence detected using fluorescence microscopy (Leica DMI 4000 B). No GFP expression was observed in controls (A1-A3), but low level GFP expression occurred in the liver for exposure to $1000 \mathrm{ng} \mathrm{E}_{2} / \mathrm{L}$ (C2) and high level GFP expression was detected in the somite muscle and heart at exposure concentrations of 100 and $1000 \mathrm{ng} \mathrm{E} / \mathrm{L}$. Weak GFP expression was also detected in the heart and somite muscle of embryos for exposure to $10 \mu \mathrm{g} / \mathrm{L} \mathrm{NP}$ (D1-D3). Embryos exposed to the higher concentrations of NP (100 $\mu \mathrm{g} / \mathrm{L})$ died. This experiment was run in duplicate and was repeated at least seven times.

oestrogens have different target tissue responsiveness and therefore potentially different health effects.

To assess the consistency of transient expression of GFP, we counted the number of GFP-positive embryos for different tissues at $72 \mathrm{hpf}$. As shown in Table 1, for controls no GFP expression was observed in the liver, heart and forebrain, but weak GFP expression signals were detected in some embryos in somite muscles $(13.3 \%)$ and the otic vesicle (16\%). In contrast, in injected embryos (72 hpf), strong GFP expression occurred in the muscle and heart on exposure to both $\mathrm{EE}_{2}$ and $E_{2}$. This was also the case for the liver in injected embryos exposed to both $\mathrm{EE}_{2}$ and $\mathrm{E}_{2}: 74 \%$ for $1000 \mathrm{ng}$ $\mathrm{EE}_{2} / \mathrm{L}, 62 \%$ for $100 \mathrm{ng} \mathrm{EE}_{2} / \mathrm{L}$ and $71 \%$ for $1000 \mathrm{ng} \mathrm{E}_{2} / \mathrm{L}$.
For the forebrain, the proportion of embryos expressing

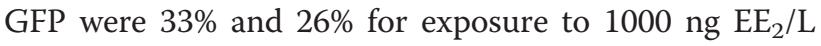
and $1000 \mathrm{ng} \mathrm{E}_{2} / \mathrm{L}$, respectively. For the NP exposure, the proportion of injected embryos showing GFP expression in muscle was $71 \%$ and $56 \%$ for the heart.

In general, the expression of GFP appeared to show a concentration-dependent response for exposure to the test oestrogens, as quantified via western blotting. The highest $\mathrm{EE}_{2}$ exposure group (1000 ng/L) indicated a 16.6-fold increase in GFP levels in whole embryos com-

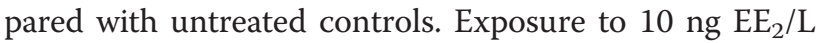
resulted in a 2.1-fold increase in GFP compared with the control (Figure 4A). Exposure to $E_{2}$ at $1000 \mathrm{ng} / \mathrm{L}$ induced a 12.4-fold increase in GFP above controls and 
A

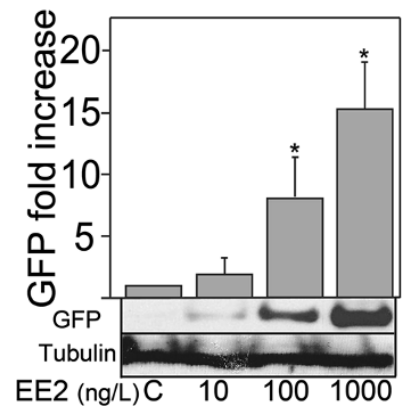

B

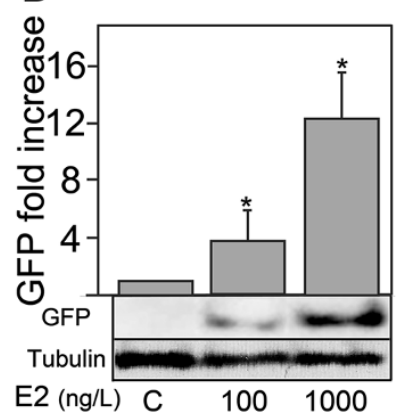

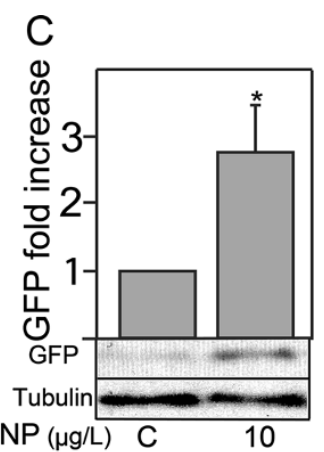

Figure 4 Western blot analysis of 72 hour old embryos injected with the mixture of the plasmid pERE-Gal4ff containing the Tol2 transposase mRNA and the plasmid pUAS-GFP. Whole-body homogenate samples of 40 embryos exposed to 17ß-oestradiol $\left(E_{2}\right)$, 17aethinyloestradiol $\left(E_{2}\right)$ and nonylphonol (NP) at various exposure concentrations (via the water) are shown. Western blot analysis showed no detectable expression of GFP (Green fluorescent protein) in controls. The level of GFP expression was induced in a concentration dependent manner for the various oestrogenic chemicals ( $E_{2}, E_{2}$ and NP). GFP expression level was quantified by using Image J (http://rsb.info.nih.gov/ij/ index/html). Error bars represent the standard deviation. This experiment was run in duplicate three times.

a 2.8-fold increase above controls for exposure to10 $\mu \mathrm{g}$ $\mathrm{NP} / \mathrm{L}$ (Figure $4 \mathrm{~B}-\mathrm{C}$ ). For the highest exposures to $\mathrm{EE}_{2}$ and $E_{2}(1000 \mathrm{ng} / \mathrm{L})$, the level of GFP induction for $E_{2}$ was stronger than for $E_{2}$, indicating that $E_{2}$ was more potent than $\mathrm{E}_{2}$.

Assessments of the relative potencies of various environmental oestrogens in our transient assay were consistent with those reported in the literature for other assays systems, with $\mathrm{EE}_{2}$ being the most potent compound compared with $E_{2}, \mathrm{NP}$ and BPA. Legler et al. (2002) [33] similarly reported that $\mathrm{EE}_{2}$ was the most potent (xeno) oestrogen compared with $\mathrm{E}_{2}, \mathrm{NP}$ and di(2-ethylhexyl) phthalate (DEHP) in their transgenic zebrafish assay. Relative potency estimates for these chemicals however, can vary depending on the assessment method. For example $\mathrm{EE}_{2}$ was shown to be 1.25 -fold more potent than $\mathrm{E}_{2}$ in yeast-based in vitro assay [53] but had around a 30 times higher potency than $E_{2}$ and $E_{1}$ for VTG induction (measured as a protein in the plasma or VTG mRNA in the liver) in female zebrafish [54] in in vivo. Thorpe et al. (2003) [55] using a VTG induction assay in vivo, reported that $\mathrm{EE}_{2}$ was between 11 to 27 times more potent than $E_{2}$. The responses to oestrogen in our zebrafish transient assay developed have shown it to be as sensitive as some other widely used oestrogen detection systems in fish (e.g. VTG induction), and as sensitive as available oestrogen responsive TG fish [35].

\section{Induction of GFP in the medaka embryo transient expression assay}

To examine if our transient assay system was applicable in other fish species, we assessed the response of GFP in the medaka embryo (Figure 5). Medaka has four types of pigment cells, namely melanophores, xanthophores, leucophores and iridophores [56] that could interfere with the detection of GFP. To avoid this, we imaged the embryos using normal light, green (GFP) and red (RFP). This was done because pigment cells are autofluorescent both in green and red spectra, and therefore by overlaying the different images (blue as background) we were able to distinguish the signal due to GFP. At 3 dpf, injected medaka embryos showed GFP expression in the skin, whereas no GFP expression was detected in the control (unexposed embryos). To enhance our ability to see clear GFP responses in other tissues, we exposed the injected embryos for 11 days. At this time, tissue specific expression became obvious in the liver, heart, gall bladder and somite (stage 40; [57]). Medaka showed a lower level of expression of GFP in muscle compared with zebrafish (See Figure. 2Ciii and Figure 5D, F) which might be due to differences in the transgenesis efficiency with Tol2 system between the two species (being lower in medaka than in zebrafish [58]). However, the different responses between the species may also be due to the physiological and genetic differences between them.

GFP expression observed in the skin and gallbladder in medaka was not observed in zebrafish, which might suggest that oestrogen receptor expression varies between fish species or oestrogen receptor binding affinities for different fish species might be different in the different embryos, at least for these organs. Differences between fish species have been shown for tissue distribution of ER subtypes as well as differences in ER affinities for environmental oestrogens ([59-61]). We do not yet know how the oestrogenic response pattern observed by GFP expression is related to the expression of ERs. The available literature suggests that ERs are expressed ubiquitously in the whole embryos both in zebrafish and medaka with an elevated expression in the neuromast cells in zebrafish [16,62]. Given this, the tissue specific response of GFP to oestrogenes may be as a consequence of the co-factors associated with ER signalling pathway. 


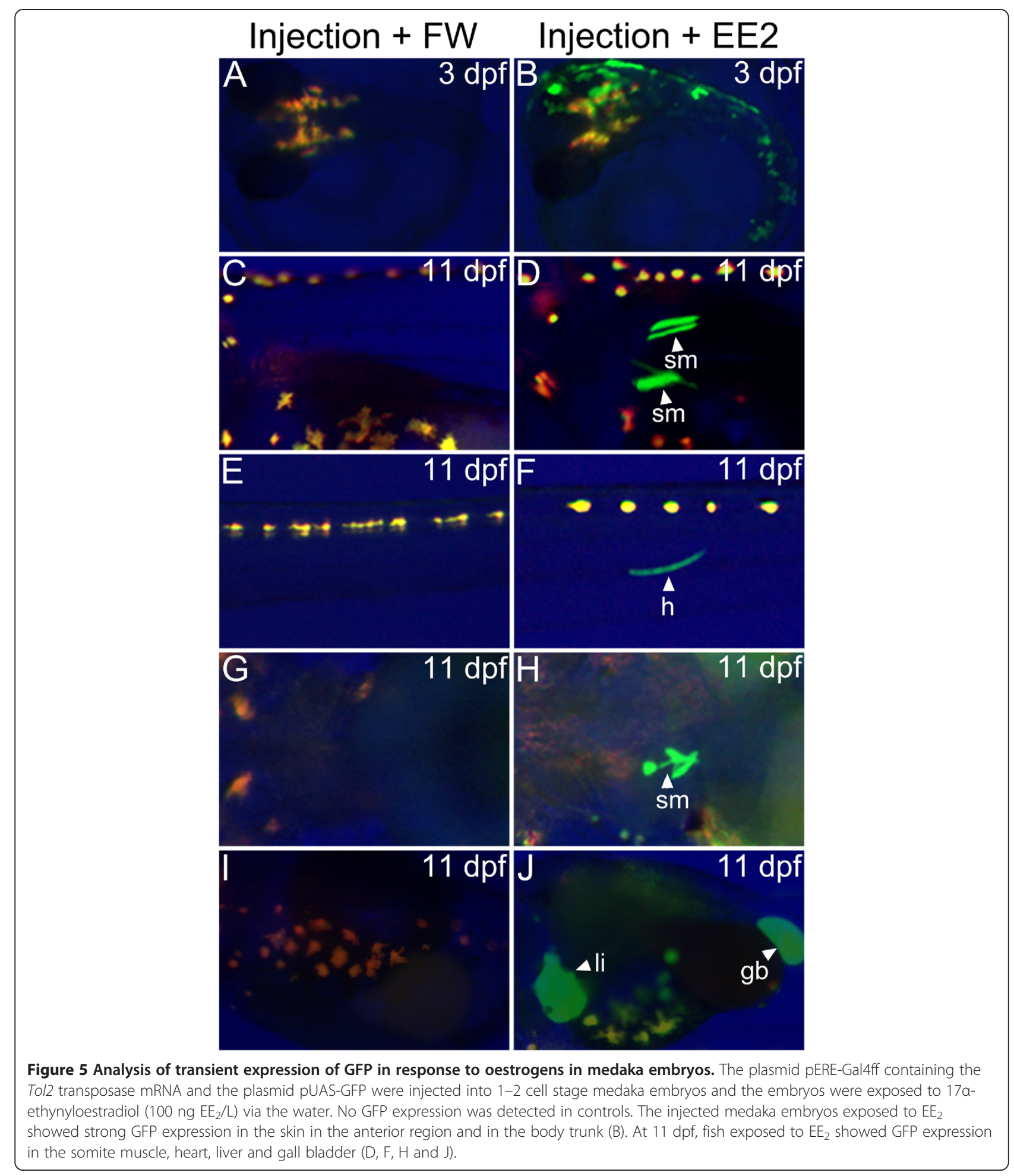

\section{Conclusion}

Despite the fact that transient expression analysis has been used in various areas of developmental biology, there has been no report using transient expression analysis for detection of oestrogen compounds. The vector system we have developed and applied in a transient assay effectively detects oestrogenic responses in body tissues. Furthermore, the transient assay system is as sensitive as many of the available TG fish for detecting oestrogens, in some cases considerably more so [63] There are however, exceptions to this, where the most sensitive TG fish developed have been shown to respond 


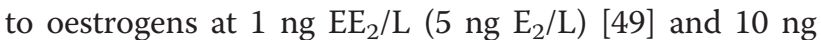

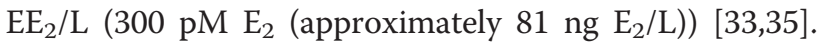
Drawbacks of the transient expression assay over TG fish includes the fact that mosaicism can make it more difficult to detect GFP expression in physically small tissues such as lateral line neuromast.

Comparing our transient assay system with VTG induction, a well established biomarker of oestrogen exposure, the detection threshold for $\mathrm{EE}_{2}$ (as an example) in zebrafish in vivo is $2 \mathrm{ng} \mathrm{EE}_{2} / \mathrm{L}$ [64]. Induction of VTG in medaka and common carp hepatocytes in culture is reported at $30 \mathrm{ng} \mathrm{E}_{2} / \mathrm{L}$ [65] and $0.01 \mu \mathrm{M} \mathrm{E} \mathrm{E}_{2}$ [66], respectively. The EC50 concentration (half maximal effective concentration) for $\mathrm{EE}_{2}$ in the MCF-7 cell assay and yeast-oestrogen screen (YES) from Folmar et al. (2002) [67] is $0.017 \mathrm{nM}(5 \mathrm{ng} / \mathrm{L})$ and $0.29 \mathrm{nM}(86 \mathrm{ng} / \mathrm{L})$, respectively.

The vector system developed detected oestrogenic EDCs in both the zebrafish and medaka, indicating the likely wide suitability for application to other fish species. This system would be a particularly powerful technique for use in fish species with long generation times and/or where there are other difficulties for generating transgenic lines in those species. The transient expression assay further provides a novel in vivo system for investigating oestrogenic effects on embryo development in fish.

\section{Methods}

\section{DNA construct for developing a transient assay}

The construct, called pTol2_3ERE-TATA-Gal4ff was based on the sequence of EREc38 (EREc38 5'-CCAGGTCAGAGTGACCTGAGCTAAAATAACACATTCAG-3') published by Sathya et al. (1997) [39]. The construct was generated by Polymerase Chain Reaction (PCR) using primers (upstream primer CCAGGTCAGAGTGACCTGAGCTAAAATAACACATTCA GCC AGGTCAGAGTG and downstream primer CTGAATGTGTTATTTTAGCTCAGG TCACTC TGACCTGGCTGAATGTGTTAT). The following conditions were used for 8 PCR cycles : denaturation at $96{ }^{\circ} \mathrm{C}$ for $1 \mathrm{~min}$, annealing at $60{ }^{\circ} \mathrm{C}$ and extension at $72{ }^{\circ} \mathrm{C}$ for $1 \mathrm{~min}$. Multiple tandem copies of EREc38 (nEREc38, where $\mathrm{n}=$ number of ERE copies) were obtained by PCR. EREc38 oligomers were prepared by restriction digestion of the 3ERE with SmaI. The reporter gene vector $\mathrm{pKS}(+)$ was digested with $\mathrm{XhoI}$ and XbaI. After obtaining the 3ERE fragment, a second fragment called TATA was constructed following the same protocol, using the following primers: (upstream primer GGCGTCGACTCTAGAGGGTATATAATAGAT CTGCGATCTAAGTAAGCTTGG downstream primer CGCGGGCCCGGCTTTACCAACAG TACCGGAATGCCAAGCTTACTTAGATCG). TATA was digested with ApaI and SalI. Gal4-ff (amplified by PCR using primers: upstream primer
GCCGGGCCCGCCACCATGAAGCTA CTGCTGTC TTCT downstream primer CGCGGTACCGATTAGTTACCCGGGAGC) was inserted into the vector which already contained the 3ERE and TATA. The BamHI and NotI fragments of p3ERE-TATA-Gal4ffwere inserted into the BamHI and NotI sites of the plasmid pBR322 vector [30], the latter containing the Tol2 transposon. The positive clones were confirmed by sequencing. The resulting plasmid was designated as pTol2_3ERE-TATA-Gal4ff.

\section{Fish maintenance and microinjection of embryos with DNA}

Zebrafish were maintained at $28 \pm 1{ }^{\circ} \mathrm{C}$ under a $14 \mathrm{~h}$ light $/ 10 \mathrm{~h}$ dark photoperiod and were fed Tetramin dry tropical flake food (Tetramin; Tetraweke, Melle, Germany) twice a day. Fertilised zebrafish eggs were collected within 20 minutes post-fertilisation. Medaka were maintained at $28{ }^{\circ} \mathrm{C}$ on a $14: 8$ light:dark cycle. All adult medaka in this study were fed Artemia twice a day. The concentration of plasmid was $20 \mathrm{pg} / \mathrm{nl}$ and $0.05 \%$ phenol red was added to make the solution visible. Under a dissecting microscope, fertilised eggs were placed in injection plate and eggs were microinjected with a syringe into the centre of the cell over the yolk-cytoplasm boundary. Embryos were microinjected at the 1-2 cell stage with $2 \mathrm{nl}$ DNA using a microinjector (INTRACEL, PICOSPRITZER $\left.{ }^{\circledR} \mathrm{III}\right)$. Some of the embryos were also exposed to various chemicals as detailed below.

\section{Chemicals}

$17 \alpha$-ethinyloestradiol ( $\geq 98 \%$ purity). $17 \beta$-oestradiol ( $98 \%$ purity), and 4-Nonylphenol (Acros Organics) were purchase from Sigma Chemical Co. Ltd. The stock solutions of $\mathrm{EE}_{2}, \mathrm{E}_{2}$ and NP were prepared in acetone and subsequently stored at $4{ }^{\circ} \mathrm{C}$, until required for the exposures. The working solutions were prepared 3 days before use in the exposures. Prior to exposure, the solvent was evaporated under a stream of nitrogen and the working solutions made up with water and stirred vigorously for 1 day. The nominal concentration of treatment solutions were: $17 \alpha$-ethinyloestradiol $\left(\mathrm{EE}_{2}\right): 1000 \mathrm{ng} / \mathrm{L}, 100 \mathrm{ng} / \mathrm{L}$ and $10 \mathrm{ng} / \mathrm{L} ; 17 \beta$-oestradiol $\left(\mathrm{E}_{2}\right): 1000 \mathrm{ng} / \mathrm{L}, 100 \mathrm{ng} / \mathrm{L}$ and $10 \mathrm{ng} / \mathrm{L} ; 4$-Nonylphenol (NP): $100 \mu \mathrm{g} / \mathrm{L}$ and $10 \mu \mathrm{g} / \mathrm{L}$ Chemicals were dissolved in embryo culture water. Fifty embryos per chemical concentration were exposed for up to 72 hours at $28{ }^{\circ} \mathrm{C}$. All experiments were run in duplicate and were repeated at least seven times.

\section{The transient expression assay using fluorescent microscopy detection}

The plasmid pTol2_ERE-TATA-Gal4ff was mixed in a 1:1 ratio with the plasmid pTol2_UAS-GFP and then mixed with transposase mRNA in a ratio of 1:2. The mixture was microinjected $(20 \mathrm{pg} / \mathrm{nl})$ into $1-2$ cell stage 
embryos of zebrafish. Injected zebrafish embryos were cultured for up to 72 hours (zebrafish) with and without exposure to $E_{2}, E_{2}$ and NP, and GFP expression examined by fluorescence microscopy (Leica DMI 4000 B). In the studies with medaka, the plasmid pTol2_ERE-TATAGal4ff with transposase mRNA was mixed in a 1:1 ratio with the plasmid pTol2_UAS-GFP and this was then injected into 1-2 cell stage medaka embryos $(20 \mathrm{pg} / \mathrm{nl})$. Injected medaka eggs were exposed to $\mathrm{EE}_{2}(100 \mathrm{ng} / \mathrm{L})$ and embryo culture water for 11 days at $28{ }^{\circ} \mathrm{C}\left( \pm 1{ }^{\circ} \mathrm{C}\right)$. GFP expression was observed under a fluorescent microscope (NIKON SMZ1500).

\section{Synthesis of transposase mRNA}

Transposase mRNA was synthesised in vitro using pCSTP which carries Tol2 transposase cDNA. The plasmid pCS-TP was digested with NotI and purified by phenol: chloroform extraction and ethanol precipitation. RNA synthesis was performed using mMessage mMachine SP6 kit (Ambion). The synthesized mRNA was purified with Quick spin column for radiolabeled RNA purification (Roche), purified by phenol: chloroform extraction and by the subsequently ethanol precipitated. The RNA precipitated was suspended in distilled water and used for co-microinjection with pTol2_ERE-TATA-Gal4ff.

\section{Western blot analysis}

Western blot analysis was employed to quantify GFP expression. Embryos were transferred to a $1.5 \mathrm{ml}$ tube filled with lysis buffer (2x LDS (Lithium dodecyl sulfate) sample buffer (Invitrogen), 2-Mercapto ethanol and distilled water), incubated at $95{ }^{\circ} \mathrm{C}$ for 5 minutes and the vessel tapped to get them to the bottom of tube. Samples were homogenised five times and centrifuged for $1 \mathrm{~min}$ at $1000 \mathrm{rpm}$. Protein samples were applied to $5 \%$ polyacrylamide-SDS gel and subjected to electrophoresis at $110 \mathrm{~V}$ for 2 hour and separated proteins were transferred to nitrocellulose membrane. The membranes were blocked for 1 hour in blocking solution (5\% skimmed milk in 1x phosphate buffered saline (PBS) $+0.1 \%$ Tween (PBSTx)), and washed three times with distilled water $\left(\mathrm{ddH}_{2} \mathrm{O}\right)$. The membranes were incubated overnight at $4{ }^{\circ} \mathrm{C}$ with primary antibody, rabbit anti-GFP (ams Biotechonology), diluted 1:2500 in blocking solution. The membranes were washed for $3 \times 15$ minutes in PBSTx. The membranes were incubated with HRP-Goat AntiRabbit IgG (Invitrogen, Carlsbad, U.S.A) at 1:2000 in blocking solution for 2 hours and then washed $3 \times 15$ minutes in PBSTx again. For detection, Western blotting luminol reagent (Thermo Scientific) was used. The intensity of GFP was analysed using Image J (http:// rsbweb.nih.gov/ij/), normalised to the intensity of alphatubulin band and indicated as fold increase in GFP over the level in control larvae.

\section{Statistical analysis}

All data are reported as mean \pm SEM. Statistical evaluation were performed using Student's $t$-test and the comparison between controls and each exposed groups. Statistical significance is indicated at the $\mathrm{p}<0.05$ level.

\section{Additional file}

Additional file 1: Figure S1 GFP expression at $4 \mathrm{dpf}$ embryos in the transient expression assay. Injected embryos without chemical exposure (A) or exposed to the oestrogenic chemicals 17a-ethinyloestradiol (1000 $\left.\mathrm{ngEE}_{2} / \mathrm{L}\right)(\mathrm{B}), 17 \beta$-oestradiol $\left(1000 \mathrm{ng} \mathrm{E}_{2} / \mathrm{L}\right)(\mathrm{C})$ and nonylphonol $(10 \mu \mathrm{gNP} / \mathrm{L})$ (D) for 4 days. Head with lateral ( $L$ ) and ventral $(V$ views (i and ii) and trunk with lateral view (iii). The shape of the liver is outlined with a white line. No GFP expression was observed in the unexposed control (A). $E_{2}$ and $E_{2}$ induced GFP expression in the heart (h), liver (li), neuromasts ( $\mathrm{n}$ ) and somite muscles (sm) (B,C). In NP exposed larvae, GFP expression was observed mainly in the muscle and heart (D).

\section{Competing interests}

The authors declare that they have no competing interests.

\section{Acknowledgements}

We thank the fish facility for fish husbandry, Koichi Kawakami for the Tol2 system. This work was co-funded by the Natural Environmental Research Council (reference NE/I014470/1) and the University of Exeter (CRT/TK). OL was funded by a studentship from Gwanglim, Korea.

\section{Authors' contributions}

All authors designed the study. OHL performed all the experiments and analysed the data. The manuscript was written by all authors $(\mathrm{OHL}, \mathrm{TK}$ and (RT). All authors read and approved the final manuscript

Received: 13 February 2012 Accepted: 24 June 2012

Published: 24 June 2012

\section{References}

1. Carlsen E, Giwercman A, Keiding N, Skakkebaek NE: Evidence fo decreasing quality of semen during past 50 years. BMJ 1992, 305 (6854):609-613.

2. Christin MS, Gendron AD, Brousseau P, Menard L, Marcogliese DJ, Cyr D Ruby S, Fournier M: Effects of agricultural pesticides on the immune system of Rana pipiens and on its resistance to parasitic infection. Environmental toxicology and chemistry/SETAC 2003, 22(5):1127-1133.

3. Jobling S, Williams R, Johnson A, Taylor A, Gross-Sorokin M, Nolan M, Tyler $C R$, van Aerle R, Santos E, Brighty G: Predicted exposures to steroid estrogens in U.K. rivers correlate with widespread sexual disruption in wild fish populations. Environ Health Perspect 2006, 114:32-39.

4. Goodhead RM, Tyler CR: Organic Pollutants--An Ecotoxicological Perspective. 2nd edition. Boca Raton, FL: C. H. Walker (Taylor \& Francis Group); 2009.

5. Brian JV, Harris CA, Scholze M, Kortenkamp A, Booy P, Lamoree M, Pojana G, Jonkers N, Marcomini A, Sumpter JP: Evidence of estrogenic mixture effects on the reproductive performance of fish. Environ Sci Technol 2007, 41(1):337-344.

6. Garcia-Reyero N, Kroll KJ, Liu L, Orlando EF, Watanabe KH, Sepulveda MS, Villeneuve DL, Perkins EJ, Ankley GT, Denslow ND: Gene expression responses in male fathead minnows exposed to binary mixtures of an estrogen and antiestrogen. BMC Genomics 2009, 10:308.

7. Filby AL, Thorpe KL, Maack G, Tyler CR: Gene expression profiles revealing the mechanisms of anti-androgen- and estrogen-induced feminization in fish. Aquatic toxicology (Amsterdam, Netherlands) 2007, 81(2):219-231.

8. Filby AL, Shears JA, Drage BE, Churchley JH, Tyler CR: Effects of advanced treatments of wastewater effluents on estrogenic and reproductive health impacts in fish. Environ Sci Technol 2010, 44(11):4348-4354.

9. Bruschweiler BJ, Wurgler FE, Fent K: Inhibitory effects of heavy metals on cytochrome P4501A induction in permanent fish hepatoma cells. Arch Environ Contam Toxicol 1996, 31(4):475-482. 
10. Chen $\mathrm{H}$, Clemens $\mathrm{TL}$, Hewison M, Adams JS: Estradiol and tamoxifen mediate rescue of the dominant-negative effects of estrogen response element-binding protein in vivo and in vitro. Endocrinology 2009, 150(5):2429-2435.

11. Mazurais D, Porter M, Lethimonier C, Le Drean G, Le Goff P, Randall C, Pakdel F, Bromage $N$, Kah O: Effects of melatonin on liver estrogen receptor and vitellogenin expression in rainbow trout: an in vitro and in vivo study. Gen Comp Endocrinol 2000, 118(2):344-353.

12. Hawkins MB, Thornton JW, Crews D, Skipper JK, Dotte A, Thomas P: Identification of a third distinct estrogen receptor and reclassification of estrogen receptors in teleosts. Proc Natl Acad Sci USA 2000, 97(20):10751-10756.

13. Kuiper GG, Lemmen JG, Carlsson B, Corton JC, Safe SH, van der Saag PT, van der Burg B, Gustafsson JA: Interaction of estrogenic chemicals and phytoestrogens with estrogen receptor beta. Endocrinology 1998, 139 (10):4252-4263.

14. Sun J, Meyers MJ, Fink BE, Rajendran R, Katzenellenbogen JA, Katzenellenbogen BS: Novel ligands that function as selective estrogens or antiestrogens for estrogen receptor-alpha or estrogen receptor-beta. Endocrinology 1999, 140(2):800-804

15. Tingaud-Sequeira A, Andre M, Forgue J, Barthe C, Babin PJ: Expression patterns of three estrogen receptor genes during zebrafish (Danio rerio) development: evidence for high expression in neuromasts. Gene Expr Patterns 2004, 4(5):561-568.

16. Froehlicher M, Liedtke A, Groh K, Lopez-Schier H, Neuhauss SC, Segner H, Eggen Rl: Estrogen receptor subtype beta2 is involved in neuromast development in zebrafish (Danio rerio) larvae. Dev Biol 2009, 330(1):32-43.

17. Lefstin JA, Yamamoto KR: Allosteric effects of DNA on transcriptional regulators. Nature 1998, 392(6679):885-888.

18. Hallare AV, Kohler HR, Triebskorn R: Developmental toxicity and stress protein responses in zebrafish embryos after exposure to diclofenac and its solvent, DMSO. Chemosphere 2004, 56(7):659-666

19. Hamm JT, Wilson BW, Hinton DE: Increasing uptake and bioactivation with development positively modulate diazinon toxicity in early life stage medaka (Oryzias latipes). Toxicol Sci 2001, 61(2):304-313.

20. Panter GH, Hutchinson TH, Hurd KS, Bamforth J, Stanley RD, Duffell S, Hargreaves A, Gimeno S, Tyler CR: Development of chronic tests for endocrine active chemicals. Part 1. An extended fish early-life stage test for oestrogenic active chemicals in the fathead minnow (Pimephales promelas). Aquatic toxicology (Amsterdam, Netherlands) 2006 77(3):279-290

21. Purdie EL, Samsudin S, Eddy FB, Codd GA: Effects of the cyanobacterial neurotoxin beta-N-methylamino-L-alanine on the early-life stage development of zebrafish (Danio rerio). Aquatic toxicology (Amsterdam, Netherlands) 2009, 95(4):279-284.

22. Higashijima S, Okamoto H, Ueno N, Hotta Y, Eguchi G: High-frequency generation of transgenic zebrafish which reliably express GFP in whole muscles or the whole body by using promoters of zebrafish origin. Dev Biol 1997, 192(2):289-299.

23. Hall C, Flores MV, Storm T, Crosier K, Crosier P: The zebrafish lysozyme C promoter drives myeloid-specific expression in transgenic fish. BMC Dev Biol 2007, 7:42.

24. Zhang $R, X u X$ : Transient and transgenic analysis of the zebrafish ventricular myosin heavy chain (vmhc) promoter: an inhibitory mechanism of ventricle-specific gene expression. Dev Dyn 2009, 238 (6):1564-1573

25. Mattingly CJ, McLachlan JA, Toscano WA Jr: Green fluorescent protein (GFP) as a marker of aryl hydrocarbon receptor (AhR) function in developing zebrafish (Danio rerio). Environ Health Perspect 2001, 109 (8):845-849.

26. Asakawa K, Kawakami K: Targeted gene expression by the Gal4-UAS system in zebrafish. Dev Growth Differ 2008, 50(6):391-399.

27. Duffy JB: GAL4 system in Drosophila: a fly geneticist's Swiss army knife. Genesis 2002, 34(1-2):1-15.

28. Hartley $\mathrm{KO}$, Nutt SL, Amaya E: Targeted gene expression in transgenic Xenopus using the binary Gal4-UAS system. Proc Natl Acad Sci USA 2002 99(3):1377-1382

29. Koga A, Suzuki M, Inagaki H, Bessho Y, Hori H: Transposable element in fish. Nature 1996, 383(6595):30
30. Kawakami K, Takeda H, Kawakami N, Kobayashi M, Matsuda N, Mishina M: A transposon-mediated gene trap approach identifies developmentally regulated genes in zebrafish. Dev Cell 2004, 7(1):133-144.

31. Kawakami K, Shima A, Kawakami N: Identification of a functional transposase of the Tol2 element, an Ac-like element from the Japanese medaka fish, and its transposition in the zebrafish germ lineage. Proc Natl Acad Sci USA 2000, 97(21):11403-11408.

32. Stuart GW, McMurray JV, Westerfield M: Replication, integration and stable germ-line transmission of foreign sequences injected into early zebrafish embryos. Development 1988, 103(2):403-412.

33. Legler J, Zeinstra LM, Schuitemaker F, Lanser PH, Bogerd J, Brouwer A, Vethaak AD, De Voogt $P$, Murk AJ, Van der Burg B: Comparison of in vivo and in vitro reporter gene assays for short-term screening of estrogenic activity. Environ Sci Technol 2002, 36(20):4410-4415.

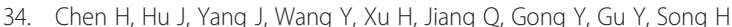
Generation of a fluorescent transgenic zebrafish for detection of environmental estrogens. Aquat Toxicol 2010, 96(1):53-61.

35. Bogers R, Mutsaerds E, Druke J, De Roode DF, Murk AJ, Van Der Burg B, Legler J: Estrogenic endpoints in fish early life-stage tests: luciferase and vitellogenin induction in estrogen-responsive transgenic zebrafish. Environmental toxicology and chemistry/SETAC 2006, 25(1):241-247.

36. Gorelick DA, Halpern ME: Visualization of estrogen receptor transcriptional activation in zebrafish. Endocrinology 2011, 152(7):2690-2703.

37. Kim DJ, Seok SH, Baek MW, Lee HY, Na YR, Park SH, Lee HK, Dutta NK, Kawakami K, Park JH: Estrogen-responsive transient expression assay using a brain aromatase-based reporter gene in zebrafish (Danio rerio). Comp Med 2009, 59(5):416-423.

38. Adam A, Bartfai R, Lele Z, Krone PH, Orban L: Heat-inducible expression of a reporter gene detected by transient assay in zebrafish. Exp Cell Res 2000, 256(1):282-290.

39. Sathya G, Li W, Klinge CM, Anolik JH, Hilf R, Bambara RA: Effects of multiple estrogen responsive elements, their spacing, and location on estrogen response of reporter genes. Mol Endocrinol 1997, 11(13):1994-2003.

40. Kurauchi K, Hirata T, Kinoshita M: Characteristics of ChgH-GFP transgenic medaka lines, an in vivo estrogenic compound detection system. Mar Pollut Bull 2008, 57(6-12):441-444.

41. Tong SK, Mouriec K, Kuo MW, Pellegrini E, Gueguen MM, Brion F, Kah O, Chung BC: A cyp19a1b-gfp (aromatase B) transgenic zebrafish line that expresses GFP in radial glial cells. Genesis 2009, 47(2):67-73.

42. Legler J, Broekhof JLM, Brouwer A, Lanser PH, Murk AJ, Van Der Saag PT, Dick Vethaak $A$, Wester $P$, Zivkovic D, Van Der Burg B: A novel in vivo bioassay for (xeno-)estrogens using transgenic zebrafish. Environ $\mathrm{SCl}$ Technol 2000, 34(20):4439-4444

43. Leclerc GM, Boockfor FR, Faught WJ, Frawley LS: Development of a destabilized firefly luciferase enzyme for measurement of gene expression. Biotechniques 2000, 29(3):590-591. 594-596, 598 passim.

44. Scholz S, Kurauchi K, Kinoshita M, Oshima Y, Ozato K, Schirmer K, Wakamatsu $Y$ : Analysis of estrogenic effects by quantification of green fluorescent protein in juvenile fish of a transgenic medaka. Environmental toxicology and chemistry/SETAC 2005, 24(10):2553-2561.

45. Ueno T, Yasumasu S, Hayashi S, luchi I: Identification of choriogenin cisregulatory elements and production of estrogen-inducible, liver-specific transgenic Medaka. Mech Dev 2004, 121(7-8):803-815.

46. Asakawa K, Suster ML, Mizusawa K, Nagayoshi S, Kotani T, Urasaki A, Kishimoto Y, Hibi M, Kawakami K: Genetic dissection of neural circuits by Tol2 transposon-mediated Gal4 gene and enhancer trapping in zebrafish. Proc Natl Acad Sci USA 2008, 105(4):1255-1260.

47. Scholz S, Gutzeit HO: 17-alpha-ethinylestradiol affects reproduction, sexual differentiation and aromatase gene expression of the medaka (Oryzias latipes). Aquatic toxicology (Amsterdam, Netherlands) 2000, 50 (4):363-373.

48. Kishida M, McLellan M, Miranda JA, Callard GV: Estrogen and xenoestrogens upregulate the brain aromatase isoform (P450aromB) and perturb markers of early development in zebrafish (Danio rerio). Comp Biochem Physiol 2001, 129(2-3):261-268.

49. Lee O, Takesono A, Tada M, Tyler CR, Kudoh T: Biosensor Zebrafish Provide New Insights into Potential Health Effects of Environmental Estrogens. Environ Health Perspect 2012, Epub Date (2012/04/19). 
50. Segner $H$, Navas JM, Schafers C, Wenzel A: Potencies of estrogenic compounds in in vitro screening assays and in life cycle tests with zebrafish in vivo. Ecotoxicol Environ Saf 2003, 54(3):315-322.

51. Bone Q, Marshall NB, Blaxter JHS: Biology of Fishes. 2nd edition. Glasgow: Blackie Academic and Professional Publishers; 1995:332.

52. Lewis SK, Lech JJ: Uptake, disposition, and persistence of nonylphenol from water in rainbow trout (Oncorhynchus mykiss). Xenobiotica; the fate of foreign compounds in biological systems 1996, 26(8):813-819.

53. Beck IC, Bruhn R, Gandrass J: Analysis of estrogenic activity in coastal surface waters of the Baltic Sea using the yeast estrogen screen. Chemosphere 2006, 63(11):1870-1878.

54. Van den Belt $K$, Berckmans $P$, Vangenechten $C$, Verheyen $R$, Witters $H$ : Comparative study on the in vitro/in vivo estrogenic potencies of 17 [beta]-estradiol, estrone, 17[alpha]-ethynylestradiol and nonylphenol. Aquat Toxicol 2004, 66(2):183-195.

55. Thorpe KL, Cummings Rl, Hutchinson TH, Scholze M, Brighty G, Sumpter JP, Tyler CR: Relative potencies and combination effects of steroidal estrogens in fish. Environ Sci Technol 2003, 37(6):1142-1149.

56. Nanda I, Kondo M, Hornung U, Asakawa S, Winkler C, Shimizu A, Shan Z, Haaf T, Shimizu N, Shima A, et al: A duplicated copy of DMRT1 in the sexdetermining region of the $\mathrm{Y}$ chromosome of the medaka, Oryzias latipes. Proc Natl Acad Sci USA 2002, 99(18):11778-11783.

57. Kinoshita M, Murata K, Naruse K, Tanaka M: A Laboratory Manual for Medaka Biology. Ames: WILEY-BACKWELL; 2009:419.

58. Kawakami K: Tol2: a versatile gene transfer vector in vertebrates. Genome Biol 2007, 8(Suppl 1):S7.

59. Lange A, Katsu Y, Miyagawa S, Ogino Y, Urushitani H, Kobayashi T, Hirai T, Shears JA, Nagae M, Yamamoto J, et al: Comparative responsiveness to natural and synthetic estrogens of fish species commonly used in the laboratory and field monitoring. Aquatic toxicology (Amsterdam, Netherlands) 2011, 109:250-258.

60. Tollefsen KE, Mathisen R, Stenersen J: Estrogen mimics bind with similar affinity and specificity to the hepatic estrogen receptor in Atlantic salmon (Salmo salar) and rainbow trout (Oncorhynchus mykiss). Gen Comp Endocrinol 2002, 126(1):14-22.

61. Menuet A, Le Page Y, Torres O, Kern L, Kah O, Pakdel F: Analysis of the estrogen regulation of the zebrafish estrogen receptor (ER) reveals distinct effects of ERalpha, ERbeta1 and ERbeta2. J Mol Endocrinol 2004, 32(3):975-986.

62. Kawahara T, Okada H, Yamashita I: Cloning and expression of genomic and complementary DNAs encoding an estrogen receptor in the medaka fish, oryzias latipes. Zoolog Sci 2000, 17(5):643-649.

63. Zeng Z, Shan T, Tong Y, Lam SH, Gong Z: Development of estrogenresponsive transgenic medaka for environmental monitoring of endocrine disrupters. Environ Sci Technol 2005, 39(22):9001-9008.

64. Orn S, Holbech H, Madsen TH, Norrgren L, Petersen Gl: Gonad development and vitellogenin production in zebrafish (Danio rerio) exposed to ethinylestradiol and methyltestosterone. Aquatic toxicology (Amsterdam, Netherlands) 2003, 65(4):397-411.

65. Nilsen BM, Berg K, Eidem JK, Kristiansen SI, Brion F, Porcher JM, Goksoyr A: Development of quantitative vitellogenin-ELISAs for fish test species used in endocrine disruptor screening. Anal Bioanal Chem 2004, 378 (3):621-633.

66. Bickley LK, Lange A, Winter MJ, Tyler CR: Evaluation of a carp primary hepatocyte culture system for screening chemicals for oestrogenic activity. Aquatic toxicology (Amsterdam, Netherlands) 2009, 94(3):195-203.

67. Folmar LC, Hemmer MJ, Denslow ND, Kroll K, Chen J, Cheek A, Richman H, Meredith $\mathrm{H}$, Grau EG: A comparison of the estrogenic potencies of estradiol, ethynylestradiol, diethylstilbestrol, nonylphenol and methoxychlor in vivo and in vitro. Aquatic toxicology (Amsterdam, Netherlands) 2002, 60(1-2):101-110.

doi:10.1186/1472-6750-12-32

Cite this article as: Lee et al:: Development of a transient expression assay for detecting environmental oestrogens in zebrafish and medaka embryos. BMC Biotechnology 2012 12:32.

\section{Submit your next manuscript to BioMed Central and take full advantage of:}

- Convenient online submission

- Thorough peer review

- No space constraints or color figure charges

- Immediate publication on acceptance

- Inclusion in PubMed, CAS, Scopus and Google Scholar

- Research which is freely available for redistribution

Submit your manuscript at www.biomedcentral.com/submit
() Biomed Central 